\title{
Infecciones respiratorias en niños con síndrome de Down
}

\author{
Dra. Marlis García', Dra. Julia Martínez², \\ Dra. Carol Rodríguez ${ }^{3}$, Dra. Wensuyen Bonilla ${ }^{4}$ \\ 1 Neumonólogo Pediatra, Hospital San Juan de Dios Caracas, Venezuela. \\ 2 Neumonólogo Pediatra, Instituto Médico la Floresta, Caracas, Venezuela. \\ 3 Neumonólogo Pediatra, Clínica Leopoldo Aguerrevere, Caracas, Venezuela. \\ 4 Neumonólogo Pediatra, Hospital San Juan de Dios, Caracas, Venezuela.
}

\section{RESPIRATORY INFECTIONS IN CHILDREN WITH DOWN SYNDROME}

\begin{abstract}
Down syndrome (DS) is the most common chromosomal abnormality among live-born infants. Respiratory tract infections are the most important cause of mortality in individuals with DS at all ages. In recent decades several studies have been performed to elucidate abnormalities of the immune system in DS. Non-immunological factors, including abnormal anatomical structures, congenital heart disease and gastro esophageal reflux, may play a role in the increased frequency of respiratory tract infections. Addressing immunological and non-immunological factors involved in the pathogenesis of infectious diseases may reduce the susceptibility to infections in DS children. Key words: Down syndrome, respiratory tract infections, immunology, innate immunity, adaptive immunity, non-immunological factors.
\end{abstract}

\section{RESUMEN}

Síndrome de Down (SD) es la anormalidad cromosómica más común entre los recién nacidos vivos. Las infecciones respiratorias son la causa más importante de mortalidad en individuos con SD en todas las edades. En las últimas décadas se han realizado varios estudios para aclarar las anormalidades del sistema inmune en SD. Factores no-inmunológicos, incluyendo estructuras anatómicas anormales, enfermedad cardíaca congénita y reflujo gastroesofágico, pueden desempeñar un papel en el aumento de la frecuencia de infecciones del tracto respiratorio. Abordar los factores inmunológicos y no inmunológicos implicados en la patogenia de las enfermedades infecciosas puede reducir la susceptibilidad a las infecciones en los niños SD.

Palabras clave: Síndrome de Down, infecciones tracto respiratorio, inmunología, inmunidad innata, inmunidad adaptativa, factores no inmunológicos.

\section{INTRODUCCIÓN}

El síndrome de Down (SD) es la anomalía cromosómica más frecuente en los niños nacidos vivos. El SD se caracteriza por una variedad de rasgos dismórficos y malformaciones congénitas que incluyen la cardiopatía congénita y la patología gastrointestinal. Pero además, el SD va asociado a un conjunto de alteraciones inmunológicas. Las infecciones de las vías respiratorias en los niños con SD son un problema frecuente que lo vemos en la práctica diaria, si bien son escasos los datos concretos en la literatura, en el siguiente artículo se realizó una amplia revisión de infecciones respiratoria en niños con $\mathrm{SD}^{(1)}$.

Correspondencia: Dra. Marlis García

Dirección Médica Hospital San Juan de Dios, Caracas-Venezuela. Calle San Juan de

Dios, Colinas de Valle arriba.

Teléfono: 005804|45402663.

E-mail: maslisgarcia@hotmail.com y marlisggarciamarquez@gmail.com

ISSN 07 |8-332| Derechos reservados.

\section{GENERALIDADES}

Las infecciones respiratorias son causa frecuente de hospitalización en menores de 2 años en países desarrollados, con cambios estacionales, en otoño e invierno; en grupos de riesgo como niños con cardiopatía congénitas (CC), prematuros, enfermedad pulmonar crónica, cromosopatías como la trisomía 2 I o el SD, deleción $22 q$ I I, entre otros ${ }^{(2-4)}$. También es frecuente que estos niños presenten diferentes grados de alteración inmunitaria, hipertensión pulmonar, incluso aunque no presenten defectos estructurales cardíacos o estos sean de poca relevancia, e hipotonía muscular. Todos estos factores los convierten en una población especialmente vulnerable a las infecciones respiratorias, esta son más frecuentes y más graves, ya que progresan fácilmente hacia una infección más severa con afección de vías bajas (bronquitis, bronquiolitis, neumonía) $)^{(5-9)}$.

Bloemers et al ${ }^{(1)}$, hacen referencia a las alteraciones del sistema inmune en los pacientes con SD que pudieran explicar la mayor incidencia de morbilidad respiratoria en estos niños. Con la finalidad de esclarecer el papel del sistema 
inmunológico en el desarrollo de estas enfermedades, en esta población concreta, sugieren algunos puntos de vista que a continuación describiremos ${ }^{(1)}$.

Los niños con SD tienen un riesgo mayor de sufrir infecciones de las vías respiratorias. Éstas se pueden dividir en infecciones de las vías respiratorias superiores (IVRS) (Ej. sinusitis, infecciones del oído medio, rinitis, amigdalitis, faringitis, laringitis subglótica) y de las infecciones de las vías respiratorias inferiores (IVRI) (Ej. neumonía, bronquiolitis), que pueden ser de origen patógeno diverso (Ej. virus, bacterias, hongos o una combinación de ellos)(1). En estos niños la incidencia de infecciones en las vías respiratorias se pueden ver favorecidos no sólo por la CC sino también por alteraciones anatómicas y fisiológicas del pulmón tales como: crecimiento pulmonar anómalo, hipoplasia alveolar, además de hipotonía generalizada que puede favorecer disfunción de la deglución y subsiguiente aspiración silenciosa ${ }^{(10,1)}$.

\section{EPIDEMIOLOGÍA}

En diferentes trabajos se pudo encontrar que la prevalencia de la neumonía adquirida en la comunidad (NAC) en los pacientes con SD ha sido evaluada en varios estudios, Pirez et al( ${ }^{(12)}$, estudiaron 697 niños hospitalizados con NAC y observó que el SD fue una de las condiciones asociadas ( 12 niños; $1,7 \%$ ), superada por las enfermedades neurológicas que afectan a la función respiratoria (29 niños; 4,2\%) y defectos congénitos del corazón (22 niños; 3,2\%). En otro estudio que involucró a 109 pacientes con SD, se observó que las infecciones respiratorias afectaban 6 I pacientes (56\%), infecciones de las vías respiratorias se produjeron con más frecuencia en niños menores de I año, la asociación con defectos congénitos del corazón en 34 pacientes (31,4\%), 19 pacientes ( 31 , 2\%) sufrieron una bronconeumonía, 6 (9,8\%) de bronquitis y 2 (3,3\%) con neumonía ${ }^{(13)}$.

La mortalidad y morbilidad, Dyce Gordon et al(13), en su estudio de las infecciones respiratorias en los niños con SD ( $n=93)$, reportó 16 muertes (I4,7\%), que en su mayoría se produjeron durante primer año de vida y fueron causadas por bronconeumonía asociada con enfermedad cardíaca congénita. No se encontraron series publicadas en las neumonías en pacientes adultos con SD.

\section{ALTERACIONES ANATÓMICAS}

Desde el punto de vista anatómico existen alteraciones en la vía respiratoria de estos niños que pueden predisponer a infecciones crónicas, entre ellas la estrechez de los conductos auditivos, lo que provoca un impacto del cerumen. La hipoplasia del macizo medio de la cara con trompas de Eustaquio más pequeñas, anormalmente insertadas y muchas veces disfuncionales, además de una superficie nasal y senos paranasales más pequeños, lo que al estar combinados podrían explicar la acumulación de líquidos en el oído medio y obstrucción del paso del aire, predisponiendo a estos pacientes a sufrir otitis media, obstrucción nasal, rinorrea y sinusitis ${ }^{(14)}$.

En las vías respiratorias inferiores se presentan patologías que son causa común de hospitalización ${ }^{(15)}$. Se ha descrito que los niños con SD tienen una incidencia mayor de sufrir un accidente pulmonar agudo y distrés respiratorio agudo cuando están ventilados mecánicamente por padecer una IVRI aguda ${ }^{(16)}$, lo que puede estar asociado a una elevación de la tasa de apoptosis de los leucocitos y células epiteliales ${ }^{(17)}$ (Tabla I).

\section{ETIOLOGÍA}

En relación a la etiología se han mencionado infecciones por microorganismos pocos frecuentes como traqueítis bacteriana por Hemophilus influenzae, neumonías por Bordetella bronchiseptica y casos graves de neumonía por Mycoplasma pneumoniae lo que rara vez ocurre en una población general(18-20). Por otro lado, el SD eleva 10 veces el riesgo de hospitalización

Tabla I. Características físicas y dismorfias en niños con síndrome de Down

Características físicas y dismorfias

Occipucio plano, braquicefalia

Fontanelas amplias y de cierre tardío

Facie plana

Ojos con fisura palpebral oblicua hacia arriba (ojos forma de almendra)

Manchas de Brushfield en iris

Epicanto

Hipertelorismo

Orejas pequeñas, oblicuas y de implantación baja

Conducto auditivo externo estrecho

Nariz pequeña y puente nasal plano

Boca abierta y lengua protruyente

Piel excesiva en la nuca y cuello corto

Manos pequeñas

Clinodactilia 5to dedo

Palma simiana (pliegue simio)

Braquidactilia

Pie en sandalia (espacio entre I er y 2 do ortejo)

Pectus excavatum o carinatum

Abdomen de aspecto abultado y diástasis de rectos anteriores

Piel seca

Piel moteada

Articulaciones laxas

Genitales externos pequeños, criptorquídea

Hipotonía

Talla baja

No todos los niños presentan todas las características físicas antes descritas y éstas pueden ser más marcadas en unos niños en comparación con otros. 
por virus sincitial respiratorio (VRS)(21), con mayor incidencia en aquellos que tienen $\mathrm{CC}^{(22)}$.

Las NAC debido a M. pneumoniae se asocia con una alta morbilidad y mortalidad en pacientes con SD, como se muestra en los casos reportados por Orlicek et al(23). Estos autores describen las características clínicas y radiológicas de la NAC en tres niños afectados con SD y que desarrollaron fiebre alta, tos productiva, escalofríos, irritabilidad y taquipnea. La radiografía $(R x)$ de tórax mostró infiltrados bilateral y los pacientes desarrollaron insuficiencia respiratoria, que requirió ingreso hospitalario. La infección por Mycoplasma en estos niños pueden ser grave porque los pacientes con $\mathrm{SD}$ presentan trastornos del sistema inmune. Los niños que tienen SD y que sufren de neumonía, se debe considerar este microorganismo como posible agente etiológico. Corretger et al.(24), publicaron un caso de un paciente con SD que desarrollo neumonía grave por $M$. pneumoniae como el agente etiológico.

También hay informes en la literatura de los casos de neumonía causada por Bordetella bronchiseptica en pacientes con SD. Se relaciona con endocarditis subaguda y tosferina leve en los niños. Los pacientes con SD que tienen inmunodeficiencia tienden a desarrollar neumonía inusual a microorganismos ${ }^{(12,24,25)}$, incluidos los agentes virales. En un estudio se describe un caso de neumonía causada por el coronavirus humano (HCoVs)-OC43 en un niño con SD y leucemia que desarrollaron fiebre y leucopenia. Tanto el recientemente descubierto HCoVs NL63 o HKUI y el prototipo HCoV OC43-y cepas HCoV-229E son considerados patógenos respiratorios en pacientes pediátricos inmunocomprometidos con SD ${ }^{(26)}$. Estos pacientes también pueden desarrollar neumonía por hongos, de acuerdo con la publicación de un caso con neumonía y derrame pleural causado por Aspergillus en un niño japonés de dos años de edad, con SD(18).

\section{RESPUESTA INMUNOLÓGICA: INNATA Y ADAPTATIVA O ADQUIRIDA}

La investigación de los últimos años han buscado esclarecer las alteraciones del sistema inmune en los pacientes con $\mathrm{SD}$. A fin de comprender como funcionan los mecanismos de defensa inmune de estos niños se plantea analizar la respuesta inmunológica innata y adaptativa de estos pacientes.

\section{DEFECTOS INMUNITARIOS EN EL SÍNDROME DE DOWN}

- Reducción leve a moderada de células T.

- Reducción leve a moderada de células B.

- Falta de la normal expansión linfocitaria en la infancia.

- El tamaño del timo es más pequeño que en controles de la misma edad.

- Reducción leve a moderada en los porcentajes de células $T$ "naive".

- Respuesta subóptima de anticuerpos a las vacunas inmunitarias.

- Reducción de inmunoglobulina A en saliva.

- Reducción de la quimiotaxis de neutrófilos.

\section{RESPUESTA INMUNE INNATA}

Primer mecanismo natural de defensa de primera línea frente a microorganismos, representado por leucocitos polimorfonucleares, células citotoxica naturales (NK), monocitos y macrófagos, además células dendríticas presentadoras de antígenos. En el SD se ha demostrado disminución de las células NK, reducción de la capacidad migratoria quimiotáctica de los leucocitos polimorfonucleares y de los fagocitos mononucleares ${ }^{(27)}$, y más constante es el aumento de la apoptosis de neutrofilos y eosinofilos en conjunto de una reducción de la capacidad citotóxica de las células NK(28).

\section{RESPUESTA INMUNE ADAPTATIVA 0 ADQUIRIDA}

En ella está implicada un mecanismo de defensa complementario desarrollado tras la exposición a un antígeno, mediado por linfocitos $\mathrm{T}$, linfocitos $\mathrm{B}$ e inmunoglobulina producidos por estos últimos. En los niños con SD hay alteraciones en el órgano que produce este tipo de células el Timo, con depleción de los timocitos corticales y posible atrofia del mismo, produciéndose una disminución en las células CDI, CD3, CD4 y CD8, disminución de la respuesta proliferativa de interleukina 4 y retraso en la maduración para formar linfocitos T, mayor los 2 primeros años de vida alterándose la proporción CD4/CD8 $8^{(29,30)}$.

Algunas investigaciones también describen disminución de los linfocitos B como consecuencia de una disfunción de su expansión y maduración ${ }^{(30)}$. Inicialmente para muchos autores las anormalidades observadas en la inmunohistología y función del timo en el SD se debía a un envejecimiento precoz del sistema inmune, sin embrago, estudios recientes apoyan la idea de que la deficiencia inmunitaria es un problema congénito de deficiencia y disregulación inmunitaria, intrínseca del SD(1).

En resumen confluyen 2 factores: las alteraciones morfológicas de las vías aéreas superiores e inferiores que facilita la penetración y permanencia de agentes patógenos y por otro lado el déficit inmunológico que reduce la capacidad de luchar y vencer a estos agentes; factores que sumados favorecen el mayor número de infecciones respiratorias que pueden prolongarse en el tiempo y originar complicaciones graves. Por ello se recomienda vacunaciones habituales contra agentes patógenos respiratorios, bacterianos y virales.

\section{RADIOLOGÍA}

No existe un patrón radiológico especifico, el mismo dependerá del agente etiológico. En un estudio se encontró dos niños evaluados por neumonía recurrente con $\mathrm{Rx}$ persistente de infiltrado. En ambos casos, las alteraciones radiológicas eran debido a hernias de Morgagni. Por lo tanto, cuando un niño con SD presenta imágenes radiográfica persistente de infiltrado, la posibilidad de defectos diafragmáticos debe ser considerado $^{(28)}$ (Figuras I y 2). 


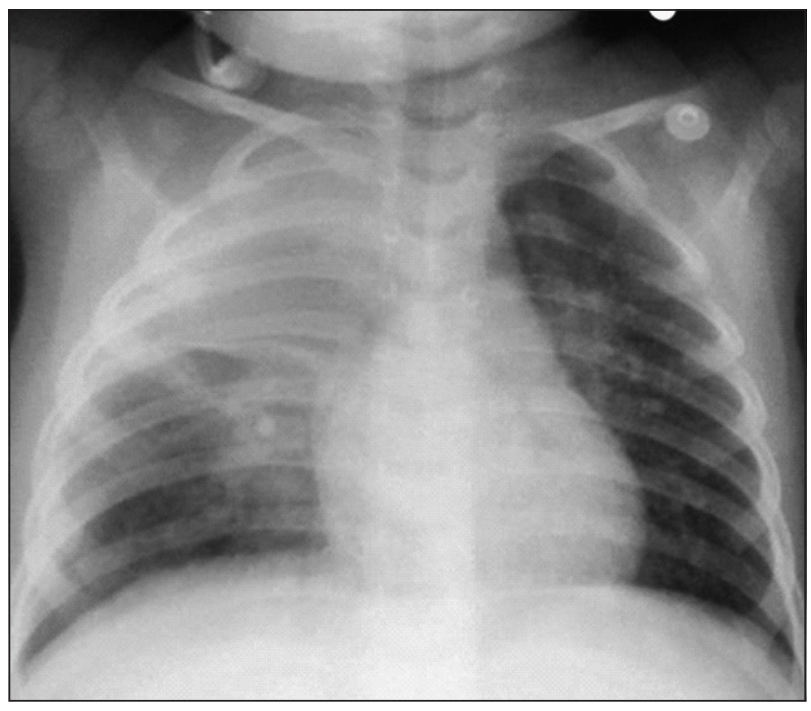

Figura I.

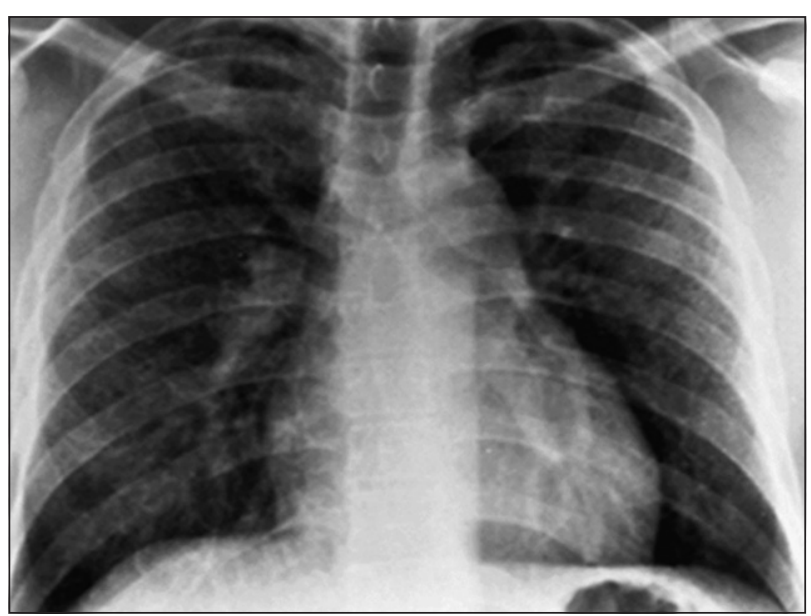

Figura 2.

\section{PREVENCIÓN}

\section{Inmunizaciones}

Los niños sanos con SD deben recibir vacunas de rutina como los otros niños. Sin embargo, las características inmunológicas y morfológicas de los niños con SD y de la gravedad y la recurrencia de estas infecciones justifican la recomendación de ciertas vacunas selectivas, que incluyen la vacunación contra el neumococo. El papel obvio causal de neumococo en estos procesos sugiere que una se debe prestar particular atención a su prevención y, por consiguiente, hay un interés en la administración de una vacuna neumocócica conjugada, incluso en los recién nacidos.

También debemos tener en cuenta la vacunación antigripal. Se han documentado anormalidades en la respuesta inmune de los niños con SD para el virus influenza. Su papel en la promoción de la otitis media y sobreinfecciones bacterianas broncopulmonares ha sido bien demostrada. El valor preventivo de la vacunación contra la influenza en estas patologías infecciosas toma especial importancia en pacientes con SD, cuya inmunogenicidad se demuestra.

La Academia Americana de Pediatría a través de su Comité de Enfermedades Infecciosas, la Asociación Española de Pediatría y el Comité de Normas de la Sociedad Española de Neonatología han elaborado recomendaciones para la prevención de las infecciones respiratorias por VRS, que es cada vez más frecuente. Debido a que parte de la población infantil con SD cae dentro de la población en riesgo, se debe considerar la adopción de medidas para prevenir esta infección, tanto higiénicas y la inmunoprofilaxis con palivizumab, que han logrado reducir el número de pacientes infectados con $\mathrm{VRS}^{(31)}$.

\section{CONCLUSIONES}

La ocurrencia frecuente de infecciones respiratorias se debe a la presencia de anormalidades estructurales y funcionales en el sistema respiratorio, lo cual es típico en niños con SD, incluyendo: La reducción del diámetro antero-posterior de la nasofaringe, que inhibe el drenaje ${ }^{(17)}$ adecuado, el desarrollo pobre de los senos paranasales y la mucosa nasal; disminución de la actividad ciliar para mantener limpia la mucosa nasal, así como la presencia de MHC, lo que predispone al niño a la congestión vascular pulmonar con posterior bronquial congestión ${ }^{(21)}$. Enfermedad cardíaca congénita se considera un factor de riesgo de infecciones respiratorias en pacientes con $\mathrm{SD}^{(12,13)}$. Weit et al. ${ }^{(32)}$, afirmó que la NAC es significativamente asociada con el asma bronquial y al reflujo gastroesofágico, en niños con SD, con historia de las infecciones respiratorias, tos productiva, y la terapia de oxígeno suplementario. En este estudio, los autores concluyeron que el efecto de la aspiración orofaríngea en el desarrollo de neumonía debe considerarse un factor de riesgo. Junto con los factores anteriormente mencionados, el retraso mental dismorfia craneofacial y que estos pacientes tienden a incrementar su probabilidad de infecciones respiratorias ${ }^{(33)}$.

\section{REFERENCIAS}

I. Bloemers BLP, Broers CJM, Bont L, Weijerman ME, Gemke RJBJ, van Furth AM. Aumento del riesgo de infecciones de las vías respiratorias en los niños con síndrome de Down: consecuencia de un sistema inmune alterado. Microbes and Infection (2010), doi: $101016 / j$.micinf.2010.05.007.

2. Law BJ, Carbonell-Estrany X, Simoes EA. An update on respiratory syncytial virus epidemiology: a developed country perspective. Respiratory Medicine 2002; 96 (SupplB): SI-7.

3. Kozak LJ, Owings MF, Hall MJ. National Hospital Discharge Survey: 2002 annual summary with detailed diagnosis and procedure data. National Centerfor Health Statistics. Vital Health Stat 2005; I3: I- 199.

4. Bonnet D, Schmaltz AA, Feltes TF. Infection by the respiratory syncytial virus in infants and young children at high risk. Cardiol Young 2005; 15: 256-65. 
5. Hilton JM, Fitzgerald DA, Cooper DM. Respiratory morbidity of hospitalized children with Trisomy 21. J Paediatr Child Health 1999; 35: 383-6.

6. Howenstine MS. Problemas pulmonares. En: Pueschel SM, Pueschel JK, editores. Síndrome de Down. Problemática biomédica. Barcelona: Masson; 1994. p. I $13-27$.

7. Chaushu S, Yefenof E, Becker A, Shapira J, Chaushu G. A link between parotid salivary $\mathrm{lg}$ level and recurrent respiratory infections in young Down's syndrome patients. Oral Microbiol Immunol 2002; 17: 172-6.

8. Shah PS, Hellmann J, Adatia I. Clinical characteristics and follow up of Down syndrome infants without congenital heart disease who presented with persistent pulmonary hypertension of newborn. J Perinat Med 2004; 32: 168-70.

9. Deerojanawong J, Chang AB, Eng PA, Robertson CF, Kemp AS. Pulmonary diseases in children with severe combined immune deficiency and DiGeorge syndrome. Pediatr Pulmonol 1997; 24: 324-30.

10. Bertrand P, Navarro H, Caussade S, Holmgren N, Sanchez J. Airway anomalies in children with Down syndrome: endoscopic findings. Pediatr Pulmonol 2003; 36.

11. Hilton JM, Fitzgerald DA, Cooper DM. Respiratory morbidity of hospitalized children with trisomy 21. J Paediatr Child Health 1999; 5: 383-6.

12. Bruijn M, van der Aa LB, van Rijn RR, Bos AP, van Woensel JB. High incidence of acute lung injury in children with Down syndrome. Intensive Care Med 2007; 33: 2179-82.

13. Dyce Gordon E, Betharte Sotomayor Y, Dyce Gordon B. Infecciones respiratorias en niños con síndrome de Down. Revista Archivo Médico de Camagüey 1997; I : 1025-55.

14. Shott SR. Down syndrome: common otolaryngologic manifestations. Am J Med Genet C Semin Med Genet 2006; I42: I31-40.

15. Brumbaugh DE, Accurso FJ. Persistent silent aspiration in a child with Trisomy 21. Curr Opin Pediatr 2002; 14: 231-3.

16. Pirez MC, Berrondo C, Giacometti M, et al. Neumonía bacteriana adquirida en la comunidad en niños hospitalizados. Arch Pediatr Urug 2003; 74: 6-I4.

17. Martin TR, Nakamura M, Matute-Bello G. The role of apoptosis in acute lung injury. Crit Care Med 2003; 31: SI84-8.

18. Fumi M, Yoshikawa, Tetsushi Y, Ayano F, et al. Pneumonia with marked pleural effusion caused by aspergillus infection. Pediatr Infect Dis J 2006; 25: 186-7.

19. Ribeiro LM, Jacob CM, Pastorino AC, et al. Evaluation of factors associated with recurrent and/or severe infections in patients with Down's syndrome. J Pediatr (Rio J) 2003; 79: I4I-8.

20. Lok RK, Harth SC, Thong YH, et al. Inmunoglobulin G subclass deficiency and predisposition to infection in Down's syndrome.
Pediatr Infect Dis J 1990; 547-5I.

21. Nelson W, Vaughan V, Mckoy J. Tratado de pediatría. 6.a ed. Barcelona: Salvat; 1975. p. I I5-7.

22. McElhinney DB, Straka M, Goldmuntz E, et al. Correlation between abnormal cardiac physical examination and echocardiographic finding in neonates with Down syndrome. Am J Med Genet 2002; I 13: 238-4I.

23. Corretger JM, Bartrons J. Neumonía grave por Mycoplasma pneumoniae en el síndrome de Down. Revista Médica Internacional sobre el Síndrome de Down 2000; 4: 33-43.

24. Jeffrey $L$, Winters $B S$, William $N$, et al. Bordetella bronchiséptica pneumonia in a patient with Down syndrome: A case report and review. Pediatrics 1992; 89: I262-5.

25. Simon A, Völz S, Fleischhack $G$, et al. Human coronavirus OC43 pneumonia in a pediatric cancer patient with Down syndrome and acute lymphoblastic leukemia. J Pediatr Hematol Oncol 2007; 29: 432-4.

26. Yasui K, Shinozaki K, Nakazawa T, Agematsu K, Komiyama A. Presenility of granulocytes in Down syndrome individuals. Am J Med.Genet 1999; 84: 406-4I2.

27. Cossarizza A, Ortolani C, Forti E, Montagnani G, Paganelli R, Zannotti $M$, et al. Age-related expansion of functionally inefficient cells with markers of natural killer activity in Down's syndrome. Blood 1991; 77: 1263-70.

28. Larocca LM, Piantelli M, Valitutti S, Castellino F, Maggiano N, Musiani P. Alterations in thymocyte subpopulations in Down's syndrome (trisomy 21). Clin. Immunol. Immunopathol 1988; 49: 175-86.

29. Roat E, Prada N, Lugli E, Nasi M, Ferraresi R, Troiano C, et al. Homeostatic cytokines and expansion of regulatory $T$ cells accompany thymic impairment in children with Down syndrome. Rejuvenation Res 2008; 11: 573-83.

30. Cossarizza A, Monti D, Montagnani G, Ortolani C, Masi M, Zannotti $M$, et al. Precocious aging of the immune system in Down syndrome: alteration of B lymphocytes, T-lymphocytes subsets, and cells with natural killer markers. Am J Med Genet suppl 1990; 7: 213-8.

31. Flórez Beledo J, Figueras Aloy J. Infecciones respiratorias por el virus respiratorio sincitial: prevención en el síndrome de Down. Revista Síndrome de Down 2006: Revista española de investigación e información sobre el Síndrome de Down. 2006; 89: 45-50.

32. Weir K, McMahon S, Barry L, et al. Oropharyngeal aspiration and pneumonia in children. Pediatr Pulmonol 2007; 42: 1024-31. Müller R, Schmidt M, Müller KM, et al. Aspiration pneumonia caused by vertebrae of a dove in a 39 year old patient with.

33. Borbolla L. Un tema de actualidad: El síndrome de Down. Rev Cubana Pediatr 1988; 60: 1078-87. 